\title{
Channel Status Prediction using Auto-regressive and Auto-regressive Integrated Predictors over WLAN Channel
}

\section{Yafei Hou*, Naoya Hokimoto and Satoshi Denno}

Graduate School of Natural Science and Technology, Okayama University, Okayama, 700-8530, Japan

\begin{abstract}
Recently, due to the increase of huge number of wireless devices such as smartphones or sensors, mobile wireless traffic is dramatically expanding each year. Cognitive radio (CR) system has been attracted attention to improve frequency usage efficiency. CR system is a technology that enables to select multiple radio systems, grasps the congestion status of communication and selects the optimum radio system. Till now, there are many researches considering the prediction of channel occupancy ratio (COR: the ration between busy duration length to resolution period $T$ ). If the start and end points of busy/idle duration from the sensing channel spectrum can be correctly predicted, it will largely benefit the wireless system design and spectrum efficiency (SE) improvement. In this paper, we will consider such research based on auto-regressive (AR) and auto-regressive integrated (ARI) models using traffic data captured from the wireless channel near a railway station. The major idea is that the busy/idle duration length can be calculated from COR value when the resolution period $T$ is short. The results confirm that our proposal can improve the prediction accuracy.
\end{abstract}

\section{Introduction}

Recently, mobile data traffic is dramatically increasing particularly in ISM band such as $2.4 \mathrm{GHz}$ and $5 \mathrm{GHz}$ bands due to the increase of huge number of smartphones and usage of IoT and M2M devices [1]. This requires the large improvement of spectrum efficiency (SE) for all ISM bands. Among all techniques, cognitive radio (CR) system has been attracted attention to improve frequency usage efficiency [2]. CR system is a technology that enables to select multiple radio systems, grasps the congestion status of wireless channel and then can select the optimum radio system. Since the usable wireless spectrum resource can be detected, predicted and rescheduled, CR system is expected to improve the current system for higher frequency utilization efficiency. The recent progress of using machine learning and data analysis on CR system has further cultivated the next generation CR system [3] To achieve efficient spectrum utilization, the channel status of all allocated frequency bands needs to be sensed and predicted which is one of the hot but challenging research topics for improving $\mathrm{SE}$ of wireless systems [4].

In CR system, spectrum sensing is a technique for appropriately detecting and predicting the channel spectrum usage status. For channel spectrum status, there are many parameters as spectrum sensing targets such as channel occupancy ratio (COR), duty circle (DC) and busy or idle duration distributions or properties so on. A tutorial paper [5] has summarized most of the existing prediction methods for the optimization of wireless resource allocation. These results have provided many efficient methods for the usage of $\mathrm{CR}$ system. However, these researches are, in the most part, considering the prediction of some key parameters such as channel occupancy ratio (COR) with a time resolution unit of either second, hour or day. There are many researches considering the prediction of channel spectrum status [6] based on measured data in some real environments. Among them, COR prediction is one of interesting research topics. COR is defined as the ratio of busy duration to the resolution $T$, it represents a measure of utilization within a specific time duration $T$. Therefore, if the prediction accuracy of COR can be improved, the channel spectrum usage status can be detected and used for decision on its usage or not according to the scheduler rules.

\section{Publication History:}

Received: December 04, 2020

Accepted: December 28, 2020

Published: December 30, 2020

\section{Keywords:}

Autoregressive predictor, Autoregressive Integrated predictor, Channel occupancy ratio prediction, Busy/idle duration prediction
There are many proposals and methods considering the channel spectrum status and COR prediction research [7-10]. A hybrid technology that combines both auto-regressive integrated moving average (ARIMA) and artificial neural networks (ANNs) is taking advantages of the unique strength of ARIMA and ANN models in linear and nonlinear modeling has been researched in [7]. Experimental results indicate that the combined model can be an effective way to improve forecasting accuracy achieved by either of the models used separately. The adaptive differential evolution (ADE) algorithm with the back propagation neural network (BPNN) can effectively improve forecasting accuracy relative to basic BPNN, ARIMA and other hybrid models which have been studied in [8]. In paper [9], a novel real time detection method based spectrum sensing technique using a logistic regression classifier, which is implemented using universal software radio peripheral (USRP) and GNU-radio, has been proposed and it can achieve a high success detection ratio of over $95 \%$ for the $2.4 \mathrm{GHz}$ ISM band. In paper [10], prediction through ML-based recurrent neural network proves to perform reasonably well, thereby it provides an accurate future spectrum occupancy information for DSA.

On the other hand, if the system can correctly predict the start and end of channel busy or idle status, the system can more efficiently utilize the available radio resources and improve spectrum efficiency when channel is accessed by a large number of devices. For example, CR system can be designed to utilize idle periods scattered in multiple frequency bands by splitting one transmission packet into small subpackets $[11,12]$ then transmitting simultaneously on the multiple bands. It can largely improve the total spectrum efficiency especially for heavy wireless traffic environments, where more spectrum

"Corresponding Author: Dr. Yafei Hou, Graduate School of Natural Science and Technology, Okayama University, Okayama, 700-8530, Japan; E-mail: yfhou@okayama-u.ac.jp

Citation: Hou Y, Hokimoto N, Denno S (2020) Channel Status Prediction using Auto-regressive and Auto-regressive Integrated Predictors over WLAN Channel. Int J Comput Softw Eng 5: 159. doi: https://doi.org/10.15344/2456-4451/2020/159

Copyright: (C) 2020 Hou et al. This is an open-access article distributed under the terms of the Creative Commons Attribution License, which permits unrestricted use, distribution, and reproduction in any medium, provided the original author and source are credited. 
Citation: Hou Y, Hokimoto N, Denno S (2020) Channel Status Prediction using Auto-regressive and Auto-regressive Integrated Predictors over WLAN Channel. Int J Comput Softw Eng 5: 159. doi: https://doi.org/10.15344/2456-4451/2020/159

Page 2 of 9

resources are required and channel status prediction is extremely important and difficult to be realized [13].

Generally, it is difficult to accurately predict the start and end points of busy/idle durations because the prediction is difficult especially for the long durations [14]. However, for some applications such as simultaneous transmission over multiple channels or bands, their time of coincidence (TOC) is short which reduces the complexity of prediction for long duration. There are many research results for COR prediction. Therefore, it will be useful for the prediction of the start and end points of status duration if their values can be calculated from the predicted COR values. When the resolution period $T$ is large, it is difficult to get the correct values because a large value $T$ will include many busy/idle durations which reduces the correctness of calculation.

In this paper, we present such research for status prediction using auto-regressive (AR) and auto-regressive integrated (ARI) models with the traffic data captured from the wireless channel in real environment. We predict the COR value using AR and ARI models and calculate the start and end timing of busy/idle duration from the predicted COR values. The major idea is that the busy and idle duration length can be calculated from COR value when the resolution period $T$ is short. The proposed method can provide the promising

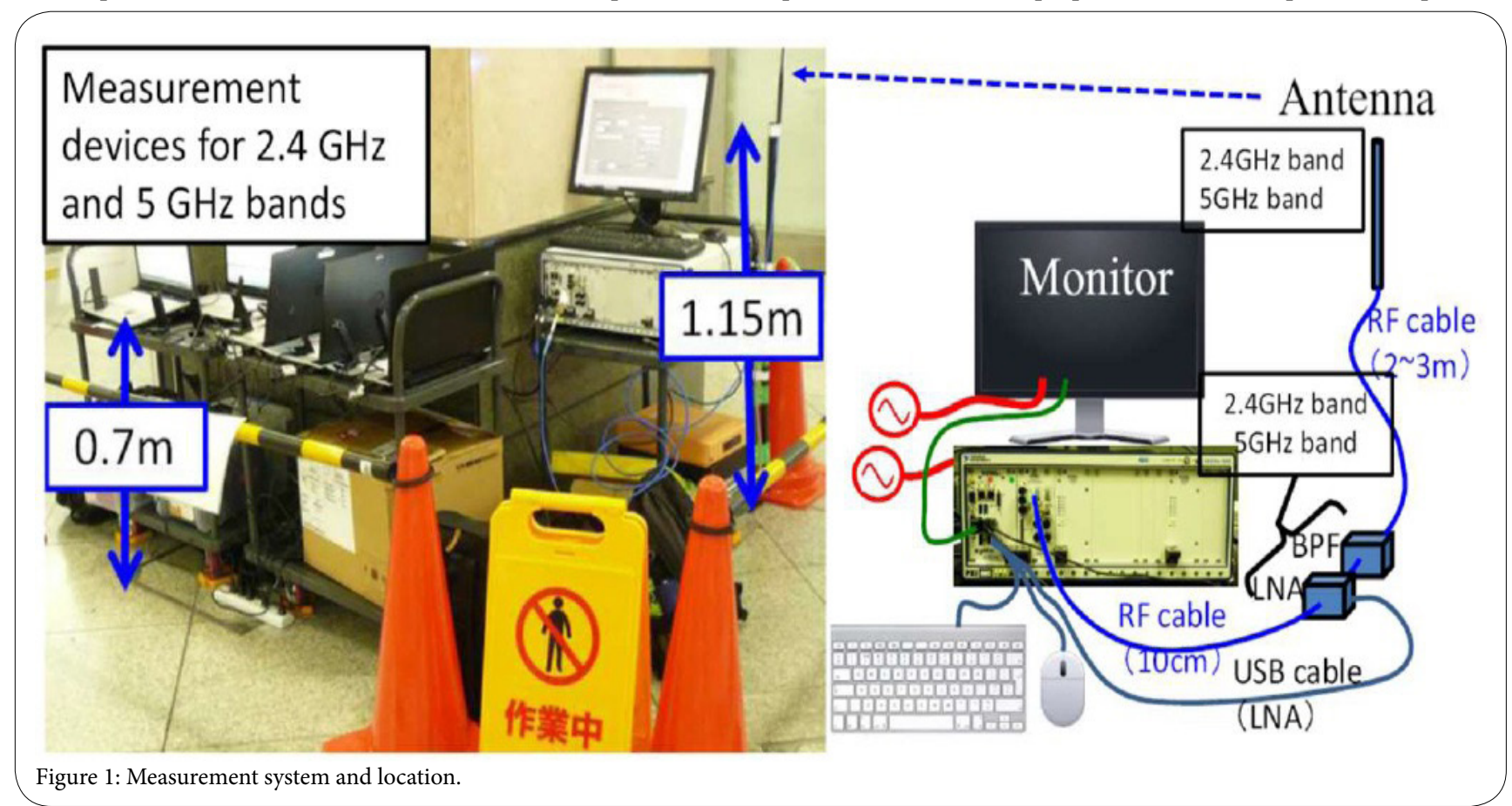

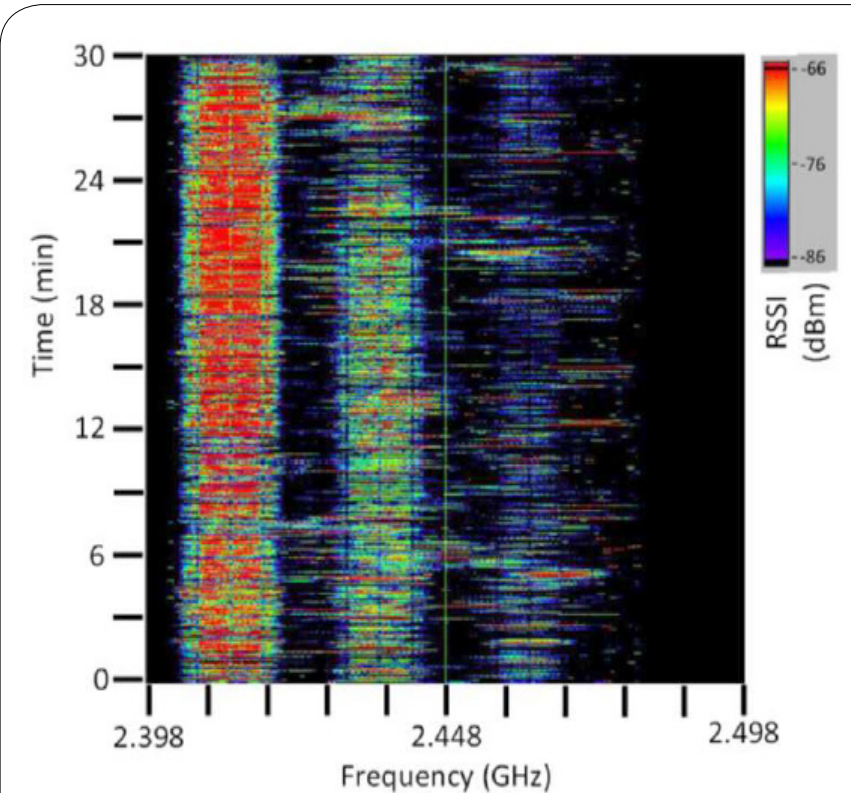

(a) $2.4 \mathrm{GHz}$ band

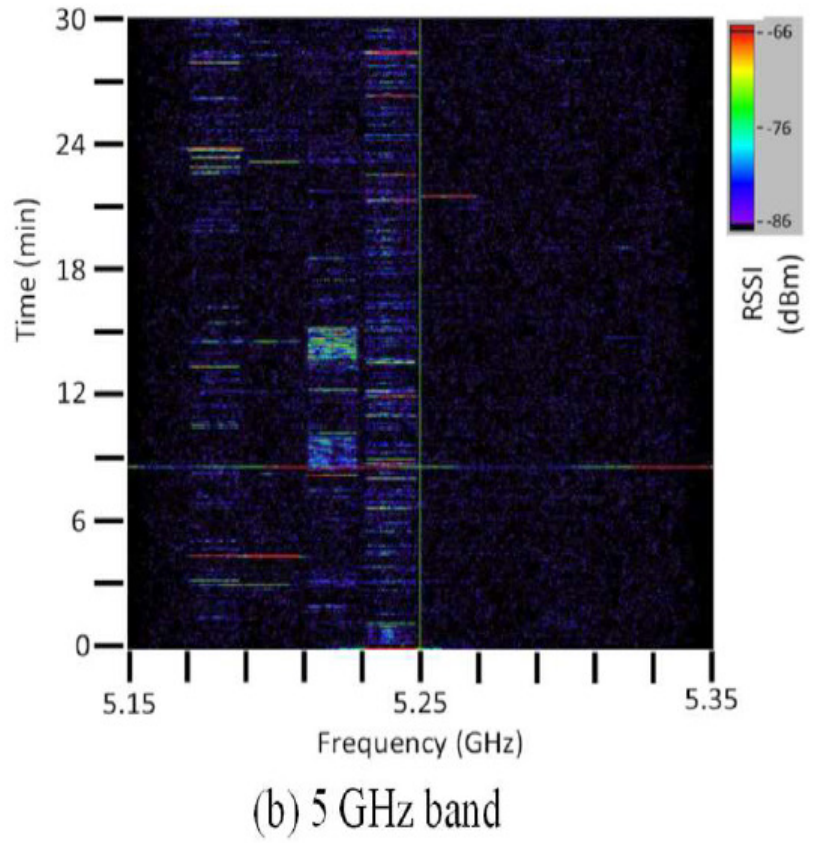

Figure 2: Spectrogram of the measured data at a railway station over $2.4 \mathrm{GHz}$ and $5 \mathrm{GHz}$ bands. 
Citation: Hou Y, Hokimoto N, Denno S (2020) Channel Status Prediction using Auto-regressive and Auto-regressive Integrated Predictors over WLAN Channel. Int J Comput Softw Eng 5: 159. doi: https://doi.org/10.15344/2456-4451/2020/159

Page 3 of 9

prediction accuracy for COR and status durations which can be used for the simultaneous transmission over multiple channels or bands.

\section{Data Measurement in Real Environment and its TOC Property}

\section{Data collection in real environment}

Measurements were carried out at a major railway station in Japan within both the 2.4 and $5 \mathrm{GHz}$ bands. Measurements were conducted at the end of January 2017. The photograph of the measurement system on location and the configuration of the measuring device are depicted in Figure 1. The measurements were operated during a rush-hour in weekday evening (busy period) and around 05:00 in the morning (non-busy period) at a major railway station for about 30 minutes.

The spectrograms of the measured data in busy period over the $2.4 \mathrm{GHz}$ and $5 \mathrm{GHz}$ bands are shown in Figure 2(a) and Figure 2(b), respectively. As shown in both figures, there are more wireless traffic over the $2.4 \mathrm{GHz}$ bands than over the $5 \mathrm{GHz}$ bands. On the other hand, even for the channels over the busy $2.4 \mathrm{GHz}$ band, some channels have more traffic than others. The results reflect that the existing channel allocation methods are not fully efficient and a new method needs to be found to mitigate the usage imbalance. The spectrograms of the measured data in non-busy period also appear the similar property.

During the measurements, we also utilized a commercial sniffing software to capture all transmitted frames on Channel 1 (2.402-2.422 $\mathrm{GHz})$ in the $2.4 \mathrm{GHz}$ band and Channel $36(5.17-5.19 \mathrm{GHz})$ in the 5 $\mathrm{GHz}$ band. From Figure 2(a) and Figure 2(b), we can find Channel and Channel 36 are one of the busiest channels in the $2.4 \mathrm{GHz}$ and $5 \mathrm{GHz}$ bands, respectively. Their header data was recorded using the software. Firstly, the frame arrival time, data-rate, and length were extracted. Then the number of data bits per symbol, bandwidth and standard (IEEE $802.11 \mathrm{~b} / \mathrm{g} / \mathrm{n}$ ) were obtained from the data-rate information based on the IEEE 802.11-2016 standards [15] using orthogonal frequency division multiplexing (OFDM). The frame

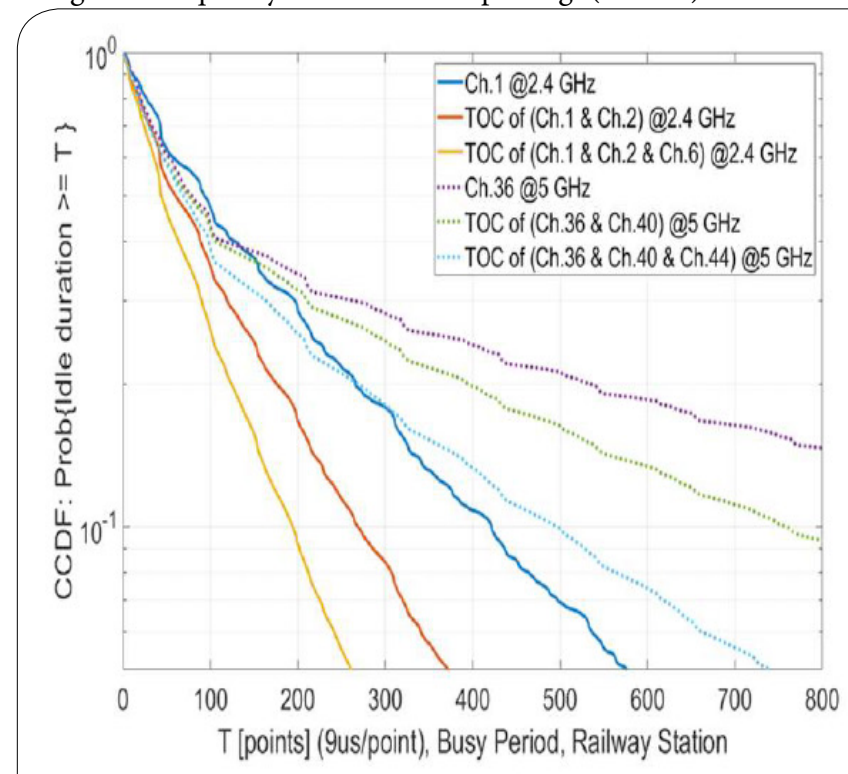

(a) Busy period

Figure 4: The time of coincidence (TOC) of multiple channels. duration was estimated from the required number of OFDM symbols after adding the MAC header with PHY preamble. The busy/ idle $(B / I)$ sequence was then generated using a granularity of $9 \mu$ ser point following the current WLAN standards.

\section{Statistics results of TOC}

For data captured in a real wireless environment, it usually includes various application data with different traffic patterns. Therefore, it is generally difficult to realize an accuracy prediction of busy/idle duration, especially when busy/idle duration is long. In this paper, we limited our consideration for the application of simultaneous transmission over multiple wireless channels or bands where one long packet can be separated into several short packets and transmitted simultaneously to one receiver $[11,12]$. This technique requires the correct prediction of idle duration of each channel or band. In addition, due to simultaneous transmission, all channels or bands must have the time of coincidence (TOC) among them. The concept of TOC is shown in Figure 3 where three channels are used for the simultaneous transmission. The TOC is the idle duration of all

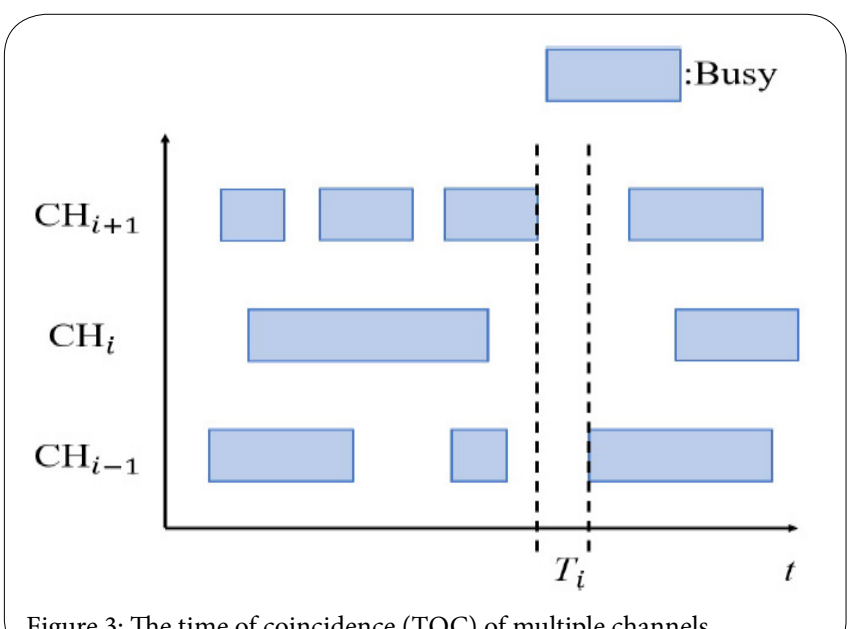

Figure 3: The time of coincidence (TOC) of multiple channels.

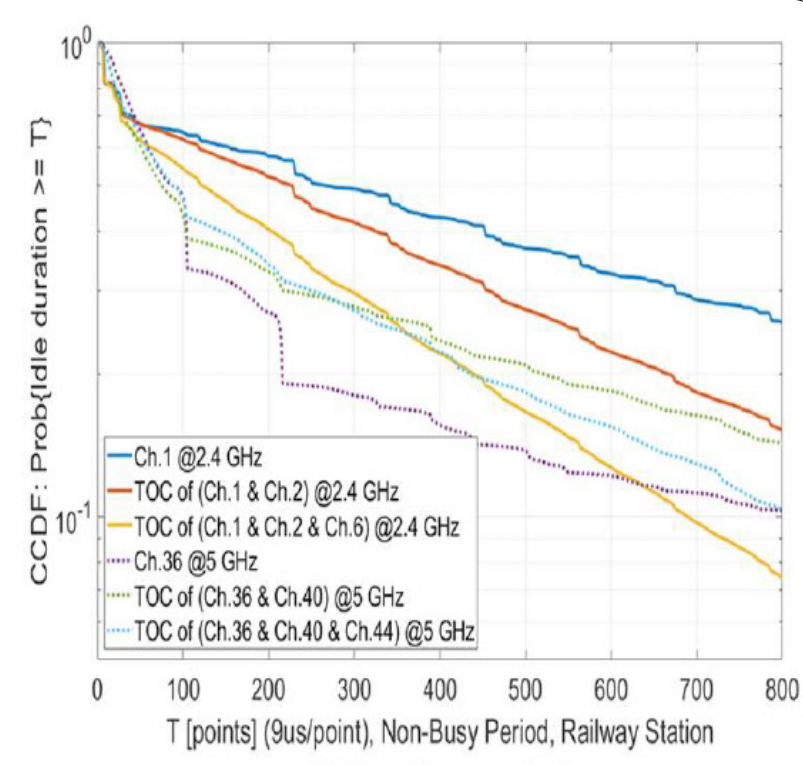

(b) Non-busy period 
Citation: Hou Y, Hokimoto N, Denno S (2020) Channel Status Prediction using Auto-regressive and Auto-regressive Integrated Predictors over WLAN Channel. Int J Comput Softw Eng 5: 159. doi: https://doi.org/10.15344/2456-4451/2020/159

Page 4 of 9

channels with the same start point and end point as shown in Figure 3 with $T_{i}$. It is easy to understand that the more channels used for simultaneous transmission, the shorter the average duration of $T_{i}$ is. This makes the prediction duration of channel status be shorter which reduces the difficulty of prediction design.

The distribution of $T_{i}$ shows the prediction length of idle duration when considering the technique of simultaneous transmission using multiple channels. We show the statistic TOC results when two or three selected channels are used for simultaneous transmission over multiple channels in Figure 4(a) and 4(b). The specific channels and location information are listed in Table 1 .

\begin{tabular}{|l|l|}
\hline Data collection location & Railway station \\
\hline Data acquisition period & Busy and Non-busy period \\
\hline Used radio channel & $\begin{array}{l}\text { Channel 1, 2,6 @ } 2.4 \mathrm{GHz} \\
\text { Channel 36, 40, } 44 \text { @ } 5 \mathrm{GHz}\end{array}$ \\
\hline
\end{tabular}

From both figures, we can find that the TOC of multiple channels decreases as the number of channels used increases. In addition, the TOC of data during non-busy period tends to be shorter than that of during busy period. For $2.4 \mathrm{GHz}$ band, TOC duration of two channels are smaller than 100 points with about $60 \%$ and $40 \%$ for busy period and non-busy period, respectively. For TOC of three channels, the idle durations will be smaller than 100 points with about $25 \%$ and $45 \%$ for busy period and non-busy period, respectively. In addition, for TOC distribution over $5 \mathrm{GHz}$ band, the TOC of idle durations is decreased with the increase of channel number. However, the TOC distribution between two channels and three channels is not largely different. The reason is that the wireless traffic over $5 \mathrm{GHz}$ band is moderate or small regardless of busy and non-busy period.

From both figures, we can find that, the idle duration of TOC is limited within one or two hundred of points for both channels over $2.4 \mathrm{GHz}$ band and $5 \mathrm{GHz}$ band regardless of busy and non-busy period. The results also show that, for the usage of simultaneous transmission over multiple channels, the status prediction needs to be have a high accuracy performance for predicting the duration of one or two hundred of points ahead.

\section{The AR/ARI Models and the Relationship between the COR and Busy/Idle Duration}

\section{AR/ARI model}

In statistics and signal processing, AR model is a representation of random process. Therefore, it is broadly used to describe certain timevarying processes in natural, economics, etc. The AR model specifies that the output variable depends linearly on its own previous and on a stochastic term (an imperfectly predictable term); thus the model is in the form of stochastic equation [16].

The notation $\operatorname{AR}(p)$ indicates an auto-regressive model with order $p$. The $\operatorname{AR}(p)$ model is defined as

$$
X_{t}=c+\sum_{i=1}^{p} \varphi_{i} X_{t-i}+\varepsilon_{t}, \quad \varepsilon_{t} \sim N\left(0, \sigma_{a}^{2}\right)
$$

where $\varphi_{1} \ldots \varphi_{p}$ are the parameters of the model, $\mathrm{c}$ is a constant and $\varepsilon_{t}$ is white noise. In some cases, for the simplification, the constant term $\mathrm{c}$ is not included for usage. Therefore, the Equation (1) becomes as

$$
X_{t}=\sum_{i=1}^{p} \varphi_{i} X_{t-i}+\varepsilon_{t}, \quad \varepsilon_{t} \sim N\left(0, \sigma_{a}^{2}\right)
$$

When using AR model for the prediction of status duration, the coefficient is calculated using training data so that they give the solution as the least squares for linear regression with Yule-Walker equation [16]. The predicted status duration $\hat{X}(n)$ can be calculated as follows

$$
\hat{X}(n)=-\sum_{i=1}^{p} \varphi_{i} X(n-i),
$$

where $\hat{X}(n)$ is the one-step predicted time-series data.

When time-series data is not a steady process, the efficient way is to take a difference operation on it and make it be a more steady one which increases the prediction performance. Let us using following equation to represent the $i$-th order difference operator on time-series data as

$$
X_{t-i}=L^{i} X_{t}
$$

Here the operator $\mathrm{L}$ is a lag for time-series data which transforms the data into one past element. For example, for one order difference operator on the input data $\mathrm{X}$, its output $\Delta X_{t}$ can be represented as

$$
\Delta^{1} X_{t} \equiv(1-L) X_{t}=X_{t}-X_{t-1}
$$

In general, the stochastic process that can be made a steady process by taking the difference operation with $d$ times is called the $d$-th order integrated process. Therefore, AR model using time-series data with $d$ order integrated process is named as ARI (auto-regressive integrated) process with $d$-order difference and represented as ARI ( $p$, $d$ ). Therefore, ARI model is just one of AR models. The whole process is identical to that of AR model but the time-series data is the one with $d$ times difference operations for the coefficients calculation.

\section{The relationship between busy/idle duration and COR}

Till now, although there are many researches on the prediction of COR, it is difficult to use for the prediction of busy/idle duration because the resolution time $T$ is long to second order which includes many busy/idle durations. However, when resolution $T$ is small to $\mu$ s order, the relationship between the COR and busy/idle duration can be easily decided. Therefore, we can employ the conventional COR prediction method for the prediction of busy/idle duration based on the relationships.

COR is the ratio of total channel occupancy duration to the resolution $T$ which is calculated as

$$
\mathrm{COR}=B_{T} / T
$$

where $B_{T}$ is total channel occupancy duration during the $T$. According to Equation (5), the COR value is ranged as in $[0,1]$.

COR value varies with the value of $T$. Therefore, the distribution of COR will be changed with different type if $T$ is changed. Figure 5 shows the COR value is changed if the resolution duration $T$ is different. Figure 6 shows the relationship between the COR and busy/ idle duration. We use Figure 5 and Figure 6 to explain our major idea of the proposal.

As shown in Figure 5, the COR value and its distribution are strongly related to the resolution duration $T$. The existed COR 
Citation: Hou Y, Hokimoto N, Denno S (2020) Channel Status Prediction using Auto-regressive and Auto-regressive Integrated Predictors over WLAN Channel. Int J Comput Softw Eng 5: 159. doi: https://doi.org/10.15344/2456-4451/2020/159

Page 5 of 9

prediction research usually utilizes a large value $T$ to get a stationary COR values for good prediction performance. However, a large $T$ will include multiple busy and idle durations which is difficult to decide the start and end points of busy/idle duration.

The relationship between the COR and the busy/idle duration is shown in Figure 6. We can divide the relationship into two types. For type 1, if COR value is 1 or 0 , it will be all busy or idle duration which can be directly decided. For some COR values among the $(0$, 1 ) as shown in Type 1 of Figure 6, using the previous COR value, either of two cases can be decided. If previous COR is not 0 , the current waveform with COR as $B_{T} / \mathrm{T}$ is the left one of the Fig. 6 with high probability. Accordingly, if the previous COR is 0 , the current waveform with COR as $B_{T} / \mathrm{T}$ is the right one of Fig. 6. However, for Type 2 where $B_{T}$ is smaller than T or multiple busy durations are included in a resolution $\mathrm{T}$, it is difficult to get the correct start or end points of busy/idle durations.

From Figure 6, we can also find that, if the resolution duration $T$ is reduced to a small value, the ratio of Type 1 will be largely increased. Although large value $T$ can get a stationary values for good prediction performance, here we use a small $T$ to find an efficient way to recover the busy/idle duration from COR using existed low-complexity predictors.
To show this, we utilize the captured data to calculate the ratio of Type 1 with the different value of $T$ as shown in Table 2 . The results are listed in Table 3(a) and 3(b). As shown in Tables 3(a) and 3(b), the ratio of Type I will be increased to more than $98 \%$ if $T$ is smaller than 25 [points] for both data captured at busy period and non-busy period. Therefore, we can obtain good prediction performance on start and end points of busy/idle duration if COR prediction can be correctly realized using small $T$.

\begin{tabular}{|c|c|c|c|c|}
\hline Data collection location & \multicolumn{4}{|c|}{ Railway station } \\
\hline Data acquisition period & \multicolumn{4}{|c|}{ Busy and non-busy period } \\
\hline Used channel & \multicolumn{4}{|c|}{ Ch.1@2.4 GHz, Ch. 36@5 GHz } \\
\hline$T$ [points] & \multicolumn{4}{|l|}{ variable } \\
\hline \multicolumn{5}{|c|}{ Table 2: Conditions for COR distribution. } \\
\hline$T$ [point] & 25 & 50 & 75 & 100 \\
\hline Busy period [\%] & 86.63 & 74.84 & 64.62 & 55.95 \\
\hline Non-busy period [\%] & 94.48 & 89.93 & 84.40 & 79.65 \\
\hline \multicolumn{5}{|c|}{ Table 3(a): The ratio of Type 1 (Ch. 1 @2.4 GHz). } \\
\hline$T$ [point] & 25 & 50 & 75 & 100 \\
\hline Busy period [\%] & 91.37 & 85.84 & 82.05 & 78.97 \\
\hline Non-busy period [\%] & 88.89 & 81.21 & 75.78 & 71.44 \\
\hline
\end{tabular}
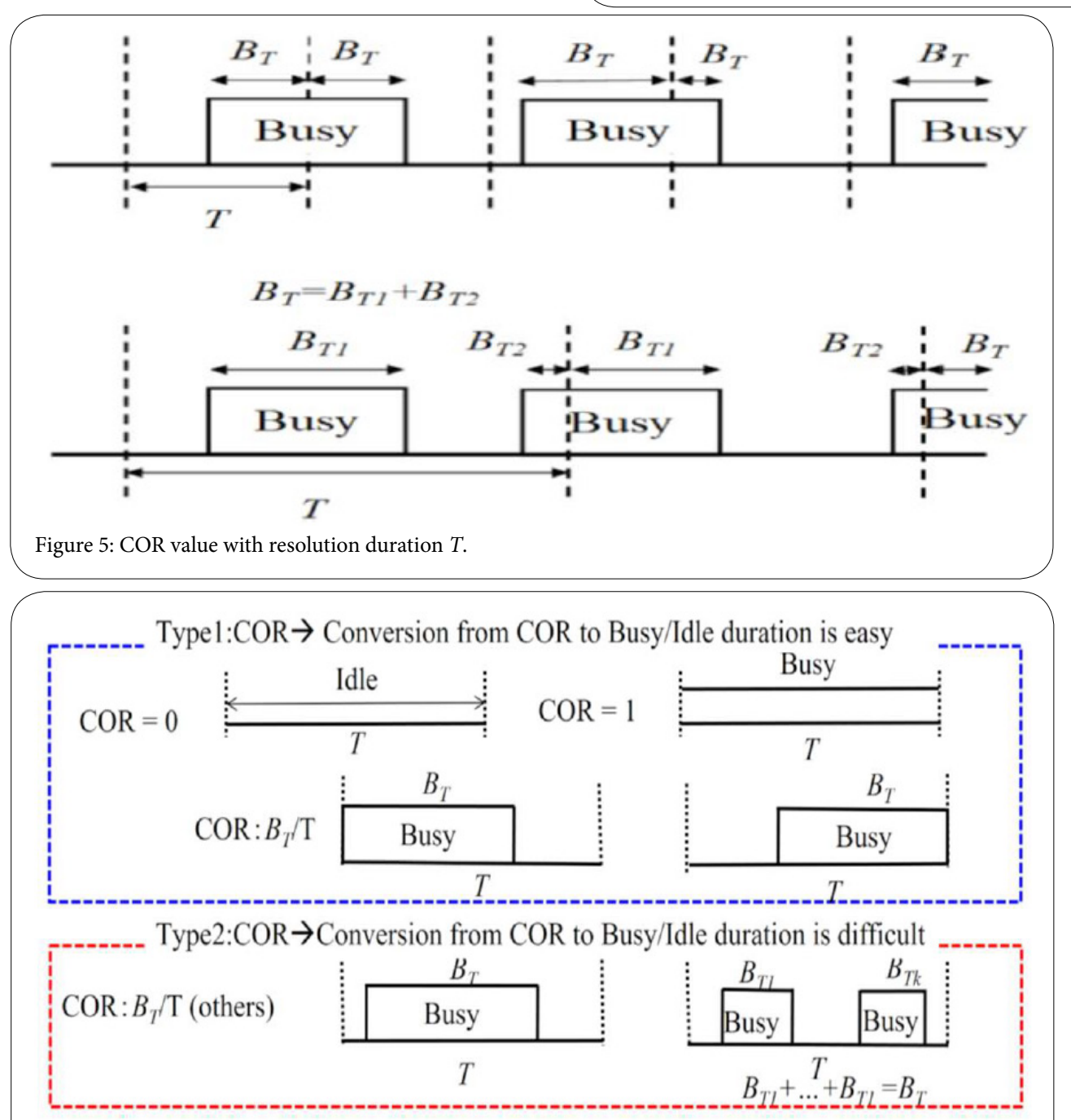

Figure 6: The relationship between the COR and busy/idle duration. 
Citation: Hou Y, Hokimoto N, Denno S (2020) Channel Status Prediction using Auto-regressive and Auto-regressive Integrated Predictors over WLAN Channel. Int J Comput Softw Eng 5: 159. doi: https://doi.org/10.15344/2456-4451/2020/159

Page 6 of 9

\section{The Performance of Prediction}

In this section, we evaluate the prediction performance of COR using AR/ARI based predictors and then the calculated idle duration. To get the parameters of AR/ARI based predictors, we use $M$ busy/ idle durations to calculate the parameters of AR or ARI predictor $\varphi_{1}, \ldots \varphi_{p}$. The calculated parameters will be same for the next $N$ times of prediction. The COR prediction error is calculated as the absolute value of prediction error which is calculated as the subtraction between the real COR value $(C O R)$ and the predicted COR value $\left(C O R_{\text {Pred }}\right)$. Table 4 shows the parameters for prediction process. For the filter polynomial or predictor order $p$ of AR and ARI predictors, we calculate different kinds of $p$ to compare their prediction accuracy. The results using $p=2$ has best accuracy than others. The prediction accuracy will be worse when we increase the value $p$. Therefore, in following prediction performance, we fix the value $p$ as 2 .

\begin{tabular}{|l|l|}
\hline Data collection location & Railway station \\
\hline Data acquisition period & busy and non-busy period \\
\hline Used channel & $\begin{array}{l}\text { Ch. 1 @2.4 GHz, Ch. 36@5 } \\
\text { GHz }\end{array}$ \\
\hline T for calculating COR [point] & $25,50,100,150,200$ \\
\hline AR/ARI predictor order (p) & 2 \\
\hline Differences order (d, ARI predictor) & $1,2,3$ \\
\hline The number of training data (M) & 100 \\
\hline The parameters used times (N) & 200 \\
\hline Table 4: Parameters for prediction algorithm.
\end{tabular}

\section{The performance of COR prediction}

Figure 7 shows the COR prediction results using AR and ARI predictors with different value of $T$ for data captured in busy period and non-busy period over $2.4 \mathrm{GHz}$ and $5 \mathrm{GHz}$ bands, respectively. Both figures show the CDF of absolute value of prediction error. For ARI predictor, we set the difference order as 1 for performance evaluation.

As shown in Figure 7, the prediction accuracy will be improved for both AR and ARI predictors with smaller value $T$ than that of large $T$. The reason is that there are many continuous $1 \mathrm{~s}$ or 0 s with small value $T$ which makes both predictors obtain good prediction accuracy. In addition, compared with AR predictor, ARI predictor can get better prediction performance than that of AR predictor especially for the data obtained from Ch. 1 at $2.4 \mathrm{GHz}$ band at busy period as shown in Figure 7(a). The reason is that the busy and idle durations variate dramatically because of the wireless access from large user terminals at $2.4 \mathrm{GHz}$ band during busy period. It can also be confirmed from the CDF of idle durations at Figure 4(a) and (b). When the scenarios are changed from large user access to small users access as from Figure 7(c) over $2.4 \mathrm{GHz}$ band during non-busy period to Figure 7(b) and (d) over $5 \mathrm{GHz}$ band during busy and non-busy period, the COR prediction difference between the AR predictor and ARI predictor is becoming small value. These results show that ARI predictor can have better COR prediction accuracy due to the difference operation of ARI when the captured data is not a steady process due to that more application patterns from more users access are included in the collected data.

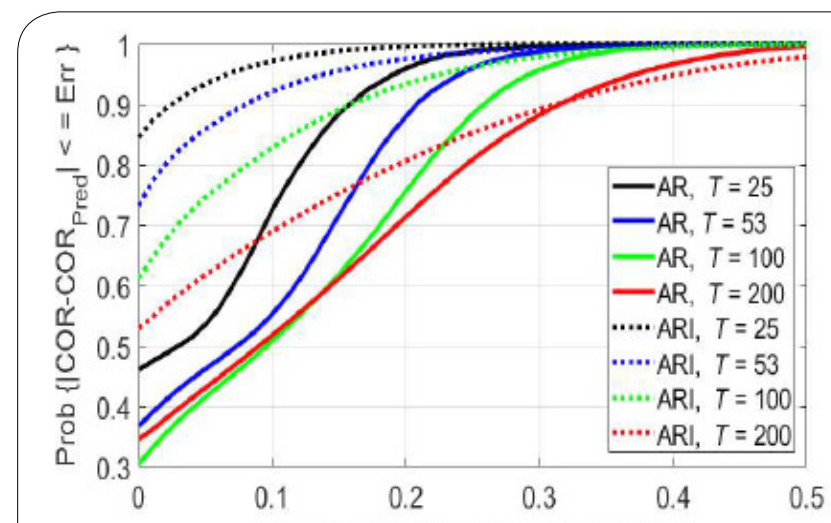

(a) Err (Busy period, Ch. $1 @ 2.4 \mathrm{GHz}$ )

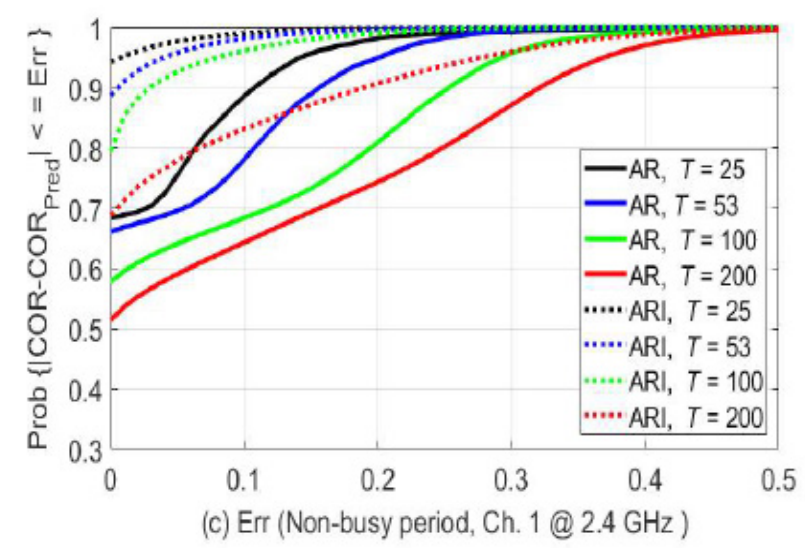

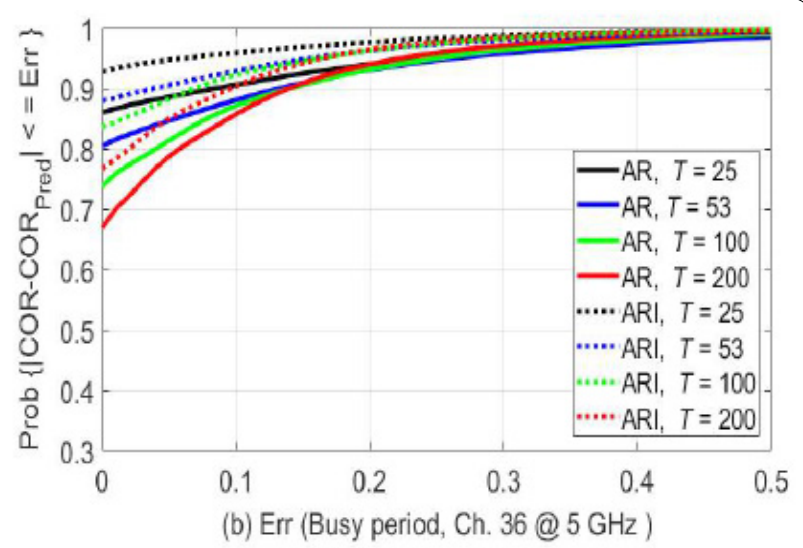

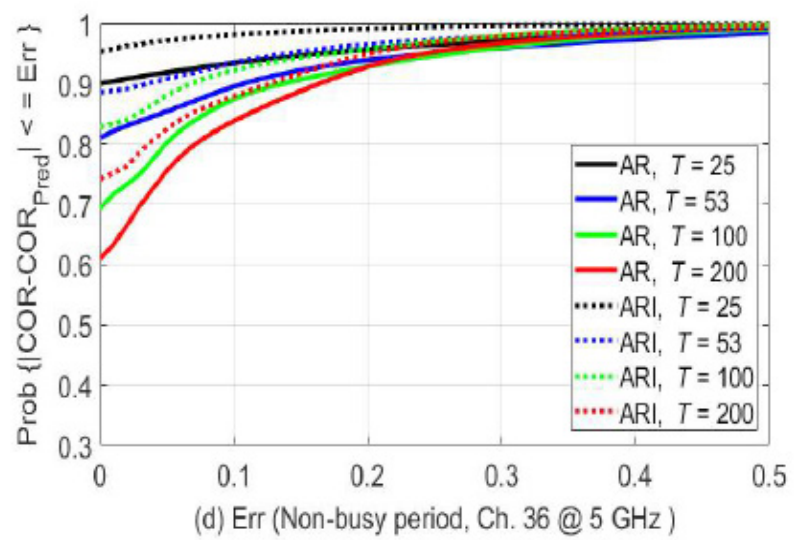

Figure 7: COR prediction performance of AR and ARI predictors (ARI: $d=1$ ). 
Citation: Hou Y, Hokimoto N, Denno S (2020) Channel Status Prediction using Auto-regressive and Auto-regressive Integrated Predictors over WLAN Channel. Int J Comput Softw Eng 5: 159. doi: https://doi.org/10.15344/2456-4451/2020/159

Page 7 of 9

To further compare the COR prediction accuracy of both predictors, we use Figure 8 to show the probability of correct prediction for both predictors using data during busy and non-busy periods with the different $T$. As shown in both figures, the COR prediction accuracy is reduced when $T$ is increased with the same reason as previous figure. In addition, the prediction accuracy of ARI predictor can be improved with about additional $40 \%$ to $50 \%$ than that of AR predictor for the data obtained from Ch. 1 at $2.4 \mathrm{GHz}$ band at busy period.

To show the prediction performance of ARI predictor with different order $d$, we use Figure 9 to compare their results with $d$ as 1,2 and 3 , respectively. As shown in the figure, ARI predictor with $d=1$ can almost get better prediction accuracy than other difference order. The reason is that high difference order will make the data varied rapidly which decreases the ARI prediction performance.

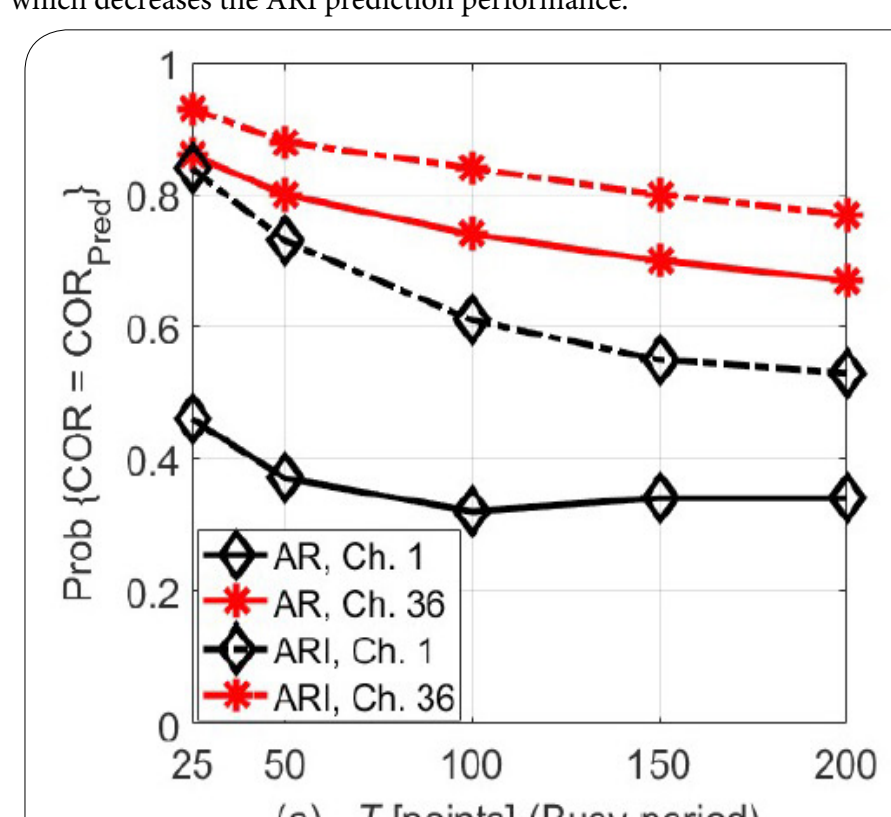

(a) $T$ [points] (Busy period)

Figure 8: COR prediction performance of AR and ARI predictors (ARI: $d=1$ ).

\section{The performance of idle duration prediction}

Finally, we show the prediction of idle duration using the COR prediction and the relation between the COR value and busy/idle duration as explained in previous section.

Here we use a new parameter named prediction success rate (PSR) to show the prediction performance between the calculated idle durations from predicted COR value and true idle durations. If the calculated idle duration is perfectly same or smaller than that of the original idle duration, we assume that prediction is successful.

Figure 10 shows the PSR value using AR and ARI predictors with different value T. As shown in Figure 10(a), for the data captured at Ch. 1 over $2.4 \mathrm{GHz}$ band during busy period, ARI predictor can

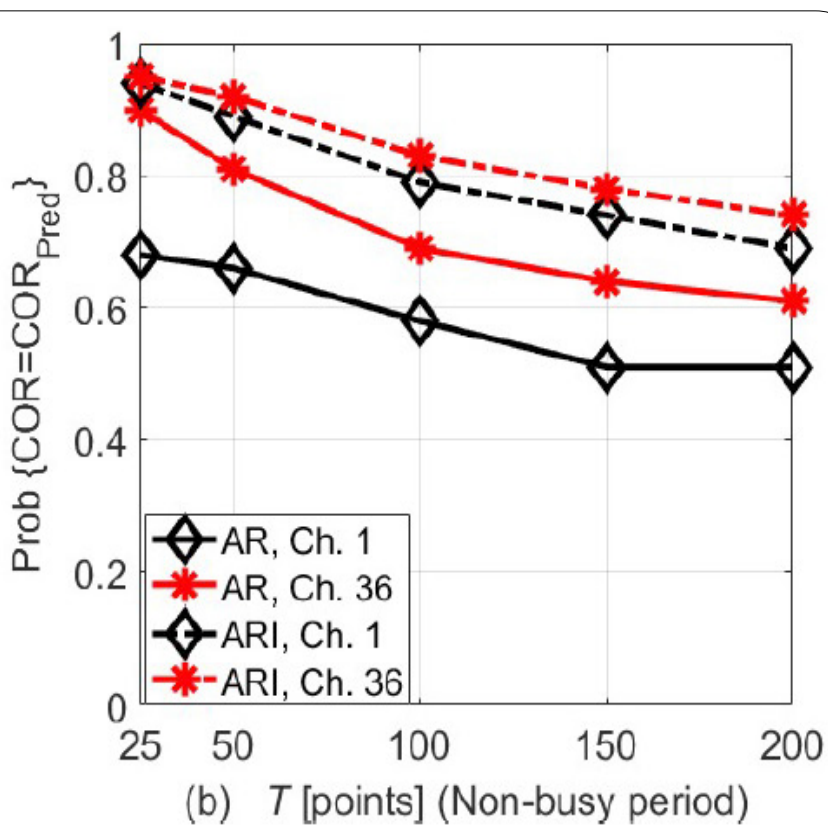

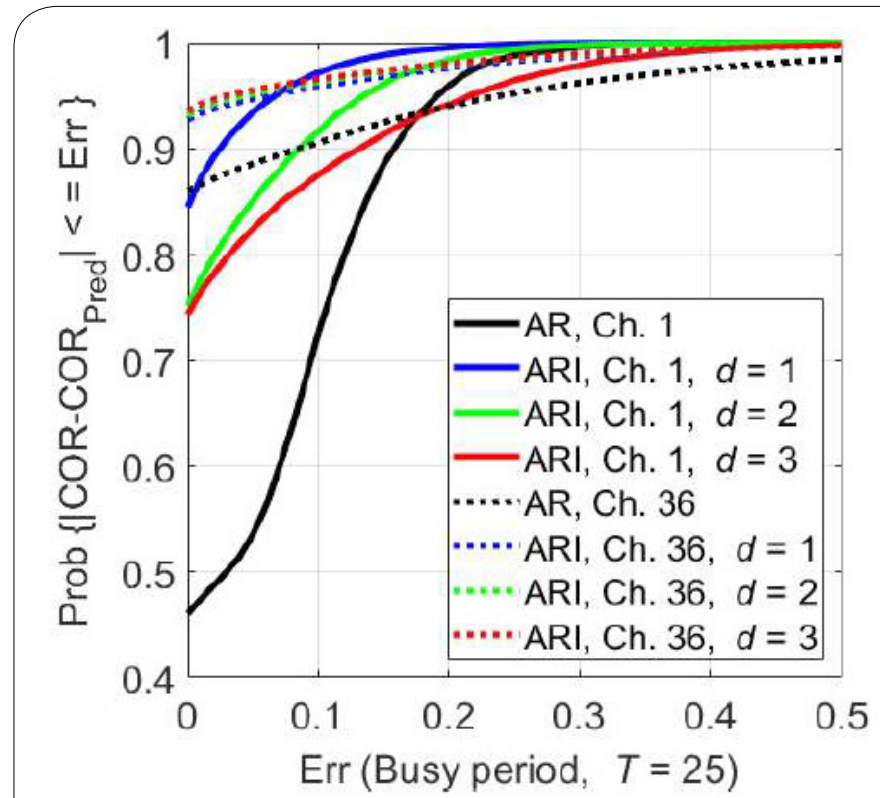

Figure 9: COR prediction accuracy with different value $d$.

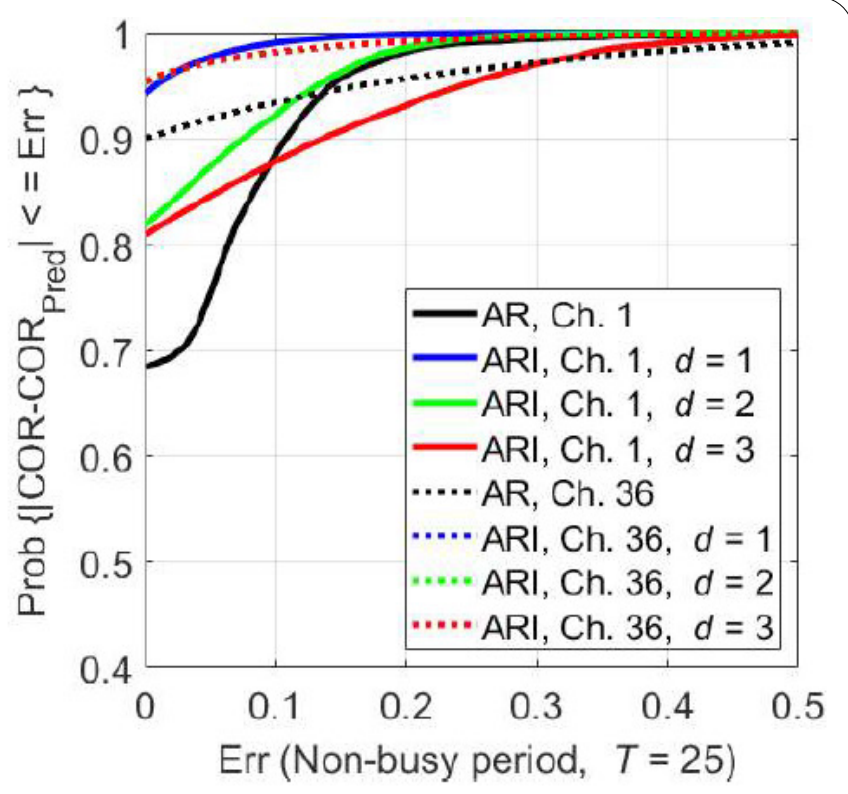


Citation: Hou Y, Hokimoto N, Denno S (2020) Channel Status Prediction using Auto-regressive and Auto-regressive Integrated Predictors over WLAN Channel. Int J Comput Softw Eng 5: 159. doi: https://doi.org/10.15344/2456-4451/2020/159

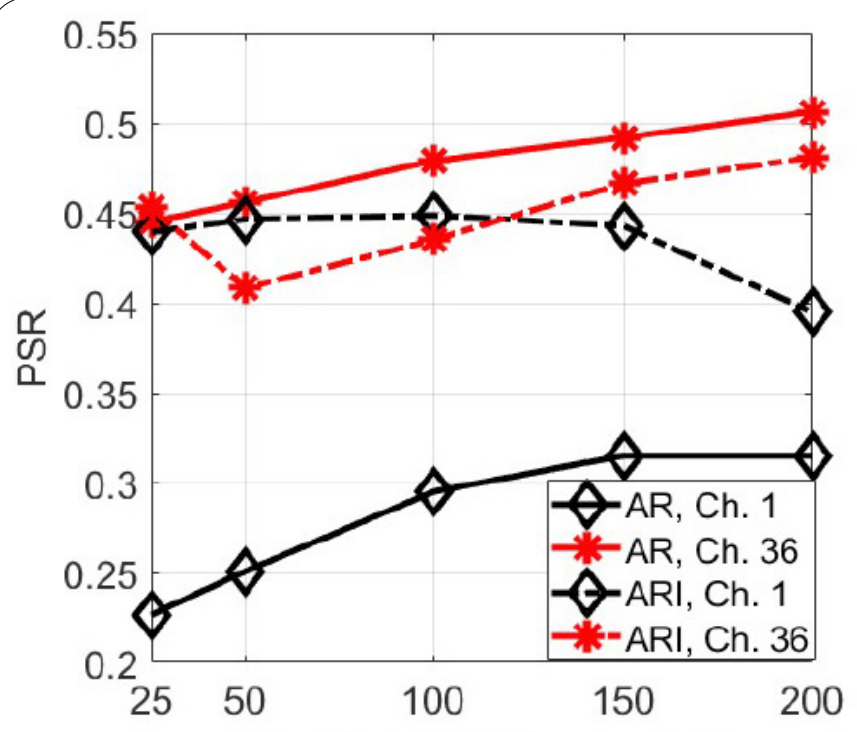

(a) $T$ [points] (Busy period)

Figure 10: The accuracy of idle duration prediction.

provide better prediction accuracy with about over $20 \%$ higher than that of AR predictor when $T$ is 25 points. Such difference of ARI and AR predictors is reduced to $8 \%$ when $T$ is increased to 200 points. For the data captured for $\mathrm{Ch} .1$ over $2.4 \mathrm{GHz}$ band during non-busy period, the accuracy improvement of ARI predictor can be $8 \%$ when $T$ is short as 25 or 50 points.

When channel has little users access such as data obtained in Ch. 36 over $5 \mathrm{GHz}$ band in both busy and non-busy periods, the PSR of that using AR predictor has better results than that of ARI predictor. The reason is that, for this scenario, the merit of COR prediction using ARI is limited compared with that of AR predictor as shown in Figure 8. In addition, ARI predictor will cause more calculation error from the predicted COR when the channel status is changed to other one. Therefore, ARI based predictor can provide better prediction accuracy than that of AR based predictor when considering the data with large wireless access from users.

On the other hand, as shown in Figure 4(a) and (b), when simultaneous transmission over multiple channels is used, the TOC or common idle duration is short with high probability when the number of channel increases. This means that for most case, it is valuable even if the prediction algorithm can provide good prediction accuracy for short idle duration. Therefore, our proposed method can be used for the usage of simultaneous transmission over multiple channels or bands.

\section{Conclusion}

In this paper, we presented the research for status prediction using $\mathrm{AR}$ and ARI predictors for the traffic data captured from the wireless channel in real environment. We predicted the COR value using AR and ARI models and calculate the start and end timing of busy/idle duration from predicted COR values. The major idea is that the busy and idle duration length can be calculated from COR value when the resolution period $T$ is short. The proposed method can provide the promising prediction accuracy for COR and status duration which can be employed for the technology of simultaneous transmission over multiple channels or bands.

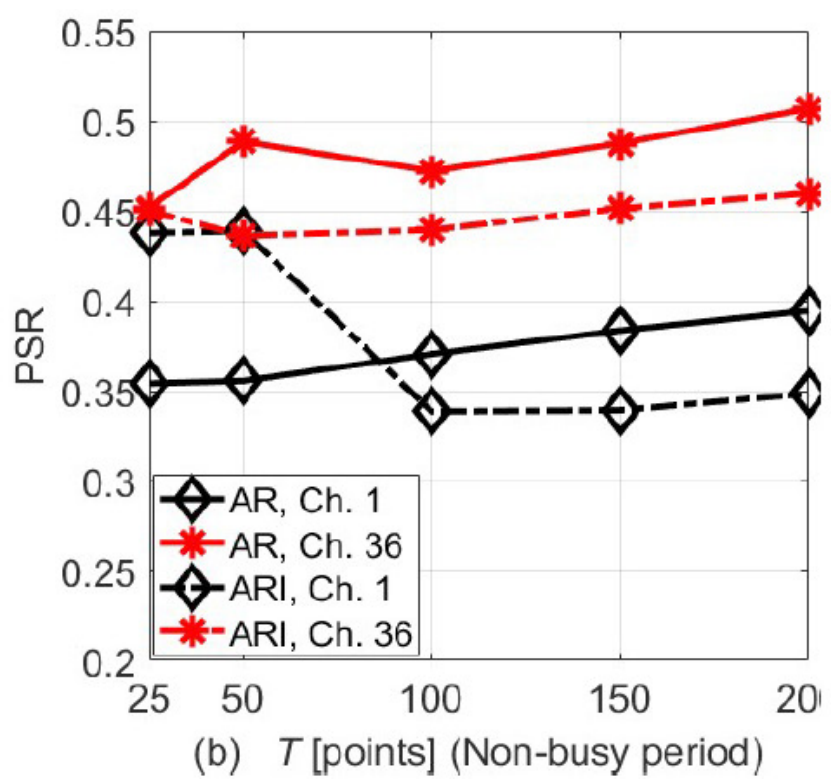

\section{Competing Interests}

The authors declare that they have no competing interests.

\section{Acknowledgment}

The data used in this paper is provided and supported by the Ministry of Internal Affairs and Communications, Japan.

\section{References}

1. Kamilaris A, Pitsillides A (2016) Mobile phone computing and the Internet of Things: a survey. IEEE Internet Things J 6: 885-898.

2. Ijaz A, Zhang L, Grau M, Mohamed A, Vural S, et al. (2016) Enabling massive IOT in $5 \mathrm{G}$ and beyond systems: PHY radio frame design considerations. IEEE Access 4: 3322-3339.

3. Struzak R (2018) Cognitive radio, spectrum, and evolutionary heuristics. IEEE Commun Mag 56: 166-171.

4. Jiang C, Zhang H, Ren Y, Han Z, Chen KC, et al. (2017) Machine learning paradigms for next-generation wireless networks. IEEE Wireless Commun 24: $98-105$.

5. Chen Y Oh HS (2016) A survey of measurement-based spectrum occupancy modeling for cognitive radios. IEEE Communications Surveys \& Tutorials 18: 848-859.

6. Song $M$, Xin C, Zhao $Y$, Cheng $X$ (2012) Dynamic spectrum access: from cognitive radio to network radio. IEEE Wireless Commun 19: 23-29.

7. Zhang GP (2003) Time series forecasting using a hybrid ARIMA and neural network model. Neurocomputing 50: 159-175.

8. Wang L, Zeng Y, Chen T (2015) Back propagation neural network with adaptive differential evolution algorithm for time series forecasting. Expert Systems with Applications 42: 855-863.

9. Srivastava S, Hashmi M, Das S, Barua D (2017) Energy detection based dynamic spectrum sensing for 2.4 GHz ISM band. International Symposium on Nanoelectronic and Information Systems, Gwalior, Madhya Pradesh, India.

10. Agarwall A, Sengar AS, Gangopadhyay R (2018) Traffic scanarios: time series versus Learning-Based Models. Journal of Communications and Information Networks 3: 44-51.

11. Fadlullah ZM, Kawamoto $Y$, Nishiyama $H$, Kato N, Egashira N, et al. (2019) Multi-hop wireless transmission in multi-band WLAN systems: Proposal and future perspective. IEEE Wireless Communications 26: 108-113. 
Citation: Hou Y, Hokimoto N, Denno S (2020) Channel Status Prediction using Auto-regressive and Auto-regressive Integrated Predictors over WLAN Channel. Int J Comput Softw Eng 5: 159. doi: https://doi.org/10.15344/2456-4451/2020/159

Page 9 of 9

12. Egashira N, Yano $K$, Sutoh $M$, Sugitani A, Amezawa $Y$, et al. (2018) Experimental evaluation of adaptive simultaneous transmission timing control considering idle/busy probability for multi-band wireless LAN. Proc of 2018 Asia-Pacific Microwave Conference.

13. Webber J, Mehbodniya A, Hou Y, Yano K, Kumagai T, et al. (2017) Study on idle slot availability prediction for WLAN using a probabilistic neural network. Proc 23rd AsiaPacific Conference on Communicatoins.

14. Ding G, Wang J, Wu Q, Yao Y, Li R, et al. (2015) On the limits of predictability in real-world radio spectrum state dynamics: From entropy theory to $5 G$ spectrum sharing. IEEE Communications Magazine 53: 178-183.

15. IEEE (2016) IEEE Standard for Information Technology - Part 11: Wireless LAN Medium Access Control (MAC) and Physical Layer (PHY) Specifications.

16. Shumway RH, Stoffer DS (2010) Time Series Analysis and Its Applications: with R Examples. Springer.

This article was originally published in a special issue:

Wireless and Mobile Networks and Their Applications

Handled by Editor:

Prof. Nobuo Funabiki

Department of Electrical and Communication Engineering

Okayama University

Japan 